\title{
Peptide-Based Inhibitors of ADAM and ADAMTS Metalloproteinases
}

\author{
Stefano Pluda ${ }^{1,2 \dagger}$, Ylenia Mazzocato ${ }^{1 \dagger}$ and Alessandro Angelini ${ }^{1,3 *}$ \\ ${ }^{1}$ Department of Molecular Sciences and Nanosystems, Ca' Foscari University of Venice, Venice, Italy, ${ }^{2}$ Fidia Farmaceutici S.p.A., \\ Abano Terme, Italy, ${ }^{3}$ European Centre for Living Technology (ECLT), Venice, Italy
}

\section{OPEN ACCESS}

Edited by:

Laura Belvisi,

University of Milan, Italy

Reviewed by:

Steven. T Whitten,

Texas State University, United States

Thomas John Brett,

Washington University in St. Louis,

United States

*Correspondence:

Alessandro Angelini

alessandro.angelini@unive.it

${ }^{t}$ These authors have contributed equally to this work and share first authorship

Specialty section:

This article was submitted to

Molecular Recognition,

a section of the journal

Frontiers in Molecular Biosciences

Received: 30 April 2021 Accepted: 30 June 2021

Published: 21 July 2021

Citation:

Pluda S, Mazzocato $Y$ and Angelini A (2021) Peptide-Based Inhibitors of ADAM and

ADAMTS Metalloproteinases. Front. Mol. Biosci. 8:703715. doi: 10.3389/fmolb.2021.703715
ADAM and ADAMTS are two large metalloproteinase families involved in numerous physiological processes, such as shedding of cell-surface protein ectodomains and extra-cellular matrix remodelling. Aberrant expression or dysregulation of ADAMs and ADAMTSs activity has been linked to several pathologies including cancer, inflammatory, neurodegenerative and cardiovascular diseases. Inhibition of ADAM and ADAMTS metalloproteinases have been attempted using various small molecules and proteinbased therapeutics, each with their advantages and disadvantages. While most of these molecular formats have already been described in detail elsewhere, this mini review focuses solely on peptide-based inhibitors, an emerging class of therapeutic molecules recently applied against some ADAM and ADAMTS members. We describe both linear and cyclic peptide-based inhibitors which have been developed using different approaches ranging from traditional medicinal chemistry and rational design strategies to novel combinatorial peptide-display technologies.

Keywords: A-disintegrin and metalloproteinase (ADAM), A-disintegrin and metalloproteinase with thrombospondin motifs (ADAMTS), metalloproteinase, peptide inhibitors, linear peptides, cyclic peptides, macrocycles

\section{INTRODUCTION}

The "A-disintegrin and metalloproteinase" (ADAM) and "A-disintegrin and metalloproteinase with thrombospondin motifs" (ADAMTS) are closely related matrix zinc-dependent metalloproteinases that belong to the adamlysin protein family (Takeda, 2016). Most ADAM metalloproteinases are membrane-anchored enzymes while the ADAMTS family comprises only secreted proteins. Both ADAM and ADAMTS proteins show a multi-domain structure and are mainly localised in the extracellular matrix (ECM) (Zhong and Khalil, 2019). Despite their structural similarities, each protein member possesses different variable domains which ensure both function and tissue specificity (Supplementary Figure 1). The domain organisation and function of each ADAM and ADAMTS protein has been extensively described elsewhere (Takeda, 2016). Briefly, ADAM proteins are responsible for shedding cell-surface protein ectodomains, such as the latent forms of growth factors, cytokines, receptors, and other molecules. Furthermore, ADAMs contribute to a wide array of biological processes, including cell adhesion, migration and signaling (Huovila et al., 2005; Seegar and Blacklow, 2019). From the twenty-one human ADAM members identified so far, only thirteen are proteolytically active (ADAM $-8,-9,-10,-12,-15,-17,-19,-20,-21,-28,-30,-33$, and -DEC1) whereas the other eight appear to be catalytically inactive (ADAM-2, $-7,-11,-18,-22$, -23, -29, and -32) (Edwards et al., 2009; Seegar and Blacklow, 2019). It has been shown that members of the latest group play important roles in development, and function as adhesion molecules rather than proteinases. However, the physiological function of the inactive ADAMs remains largely unknown. 
Unlike ADAMs, all ADAMTS proteins are catalytically active and contain a varying number of C-terminal thrombospondin type-1 (TSP-1) motifs instead of the ADAM transmembrane and cytoplasmic domains (Apte, 2020). ADAMTSs participate in ECM maintenance, tissue morphogenesis and remodeling by cleaving a large number of matrix proteins (Kelwick et al., 2015; Apte, 2020). ADAMTS family consists of nineteen members that can be sub-classified according to their known substrates, namely aggrecanases or proteoglycanases (ADAMTS-1, $-4,-5,-8,-9,-15$ and -20 ), procollagen N-propeptidases (ADAMTS-2, -3 and -14), cartilage oligomeric matrix protein (also known as thrombospondin-5) cleaving proteinases (ADAMTS-7 and -12), von Willebrand factor (VWF) cleaving proteinase (ADAMTS-13) and a group of orphan enzymes (ADAMTS-6, -10, -16, -17, -18 and -19) (Kelwick et al., 2015; Apte, 2020).

Aberrant expression or dysregulation of ADAMs and ADAMTSs activity has been linked to the development of cancer (Sun et al., 2015; Jackson et al., 2017) and numerous inflammatory (Lambrecht et al., 2018; Mead and Apte, 2018), neurodegenerative (Duffy et al., 2009; Lemarchant et al., 2013) and cardiovascular (Zhong and Khalil, 2019; Santamaria and de Groot, 2020) diseases to name but a few. Hence, ADAM and ADAMTS proteins represent important drug targets for the prevention and treatment of several human diseases.

Inhibition of ADAM and ADAMTS metalloproteinases have been attempted using various small molecules. The majority of these molecules bear either the hydroxamate, carboxylate, thiolate, tartrate, phosphinate, thiadiazole, hydroxyquinoline or imida-zolidine-2,4-diones groups, which are capable of competitively binding the zinc ion present in the catalytic site of the metalloproteinase (Moss et al., 2001; Georgiadis and Yiotakis, 2008; Yiotakis and Dive, 2009). In addition, inhibitory molecules lacking a zinc-binding moiety have also been reported (Gilbert et al., 2011). Despite the different approaches attempted, these conventional small moleculebased inhibitors have mostly had limited success in the clinic (Georgiadis and Yiotakis, 2008; Moss and Minond, 2017). Failures have often been attributed to off-target effects due to structural similarities among the active sites of the different metalloproteinases and the consequent toxicities associated (Supplementary Figure 2) (Georgiadis and Yiotakis, 2008; Tortorella et al., 2009; Raeeszadeh-Sarmazdeh et al., 2020). As a result, there is a great interest in developing novel ADAM and ADAMTS inhibitors that can selectively target a single member of each family. Efforts to generate more effective therapies have led to the development of protein-based inhibitors such as monoclonal antibodies and tissue inhibitors of metalloproteinases (TIMPs) which are currently being tested in advanced clinical trials (Santamaria and de Groot, 2019; Raeeszadeh-Sarmazdeh et al., 2020). Unlike small moleculebased inhibitors, protein-based therapeutics offer a higher selectivity due to a larger surface of interaction and therefore, reduced toxicity. Indeed, most protein-based inhibitors do not bind the active site of the ADAM and ADAMTS enzymes but recognise surface-exposed loops that are poorly conserved between closely related family members. Inhibition appears to occur through a variety of mechanisms including i) binding at or near the active site to block substrate access (direct manner) or ii) binding to regions that are allosterically linked to the active site region (indirect manner) (Wu et al., 2007; RaeeszadehSarmazdeh et al., 2020). A major drawback of protein-based therapeutics compared to small molecule inhibitors is that they are not orally available and therefore need to be injected either subcutaneously or intravenously.

While most of these small-molecules and protein-based inhibitors have been thoroughly described elsewhere (Moss et al., 2001; Georgiadis and Yiotakis, 2008; Yiotakis and Dive, 2009; Murumkar et al., 2010; Gilbert et al., 2011; El Bakali et al., 2014; Santamaria et al., 2017; Malemud, 2019) this mini review focuses exclusively on peptide-based inhibitors, an alternative and emerging type of ADAMs and ADAMTSs metalloproteinase inhibitors. Similar to the protein-based inhibitors, peptides inhibitors are capable of binding the target with a surface of interaction large enough to obtain high efficiency and selectivity (Pelay-Gimeno et al., 2015; Atangcho et al., 2019). Like small molecules, peptide-based inhibitors can be synthesised chemically, possess ease of modification, low toxicity, and reduced antigenicity. Their modular structure and the commercial availability of hundreds of amino acid building blocks simplifies the rapid development of peptides with tailored properties (Rastogi et al., 2018; Muttenthaler et al., 2021). Here, we will mention examples of both linear and cyclic peptide-based inhibitors and the different approaches undertaken for their development will be described.

\section{PEPTIDE-BASED INHIBITORS OF ADAMs}

To date, peptide-based inhibitors have been successfully developed against only two members of the ADAM family: ADAM-8 and ADAM-17 (Figure 1 and Supplementary Table 1). The latter one, also known as tumor necrosis factora converting enzyme (TACE), is involved in shedding the proinflammatory cytokine tumor necrosis factor-a (TNF- $\alpha$ ) at the cell surface (Zunke and Rose-John, 2017). Altered activity of ADAM-17 has been associated with the onset of numerous inflammatory diseases, such as cardiac hypertrophy, arthritis, Chron's disease and cancer (Feldmann and Maini, 2008; Moss and Minond, 2017; Lambrecht et al., 2018). The first peptidebased inhibitors targeting ADAM-17 were identified using synthetic combinatorial libraries of seven amino acids-long peptidomimetics (Hu et al., 2005a; Hu et al., 2005b). Libraries were designed to mimic the sequence of the cleavage sites in denatured collagen type II and include zinc-ion chelating sidegroups. The screening revealed two low micromolar inhibitors, named regasepin 1 (Figure $\mathbf{1 A}$ ) and regasepin 2, that inhibit related metalloproteinases MMP-8 and MMP-9 with similar potency (Supplementary Table 1; (Hu et al., 2005b; Dillen et al., 2006)). Further structure-based optimisation of regasepin 1 led to the generation of a small peptide repertoire bearing different $\mathrm{D}$-form amino acids in place of $\mathrm{Val}$ and Lys in position $\mathrm{P}^{\prime}$ at $\mathrm{P} 2^{\prime}$, respectively. The best selected peptide, named 


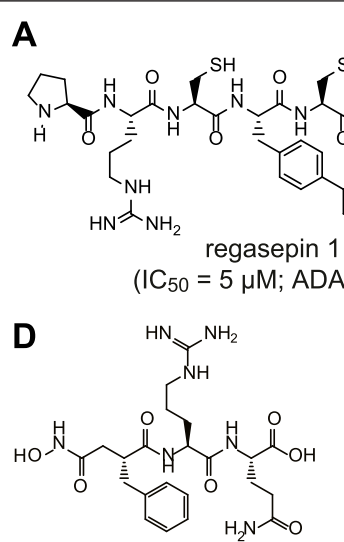

Hxm-Phe-Arg-GIn $\left(K_{\mathrm{i}}=47 \mathrm{nM} ;\right.$ ADAM-17)

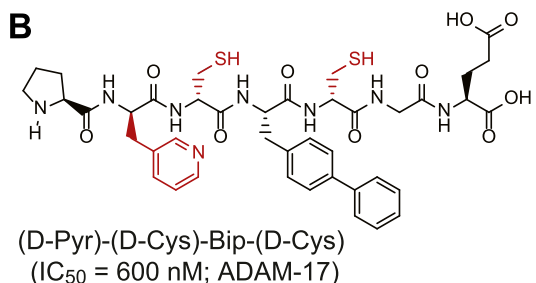

$\left(\mathrm{IC}_{50}=600 \mathrm{nM} ;\right.$ ADAM-17)

F

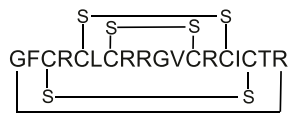

RTD-1

G

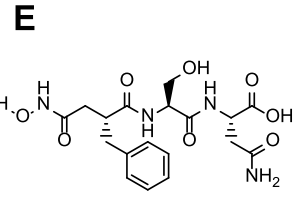

Hxm-Phe-Ser-Asn $\left(K_{\mathrm{i}}=92 \mathrm{nM} ; \mathrm{ADAM}-17\right)$

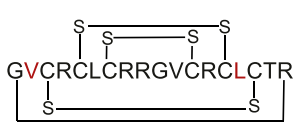

RTD-2

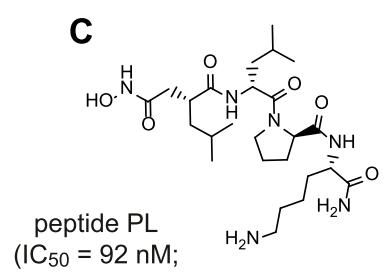

ADAM-17)

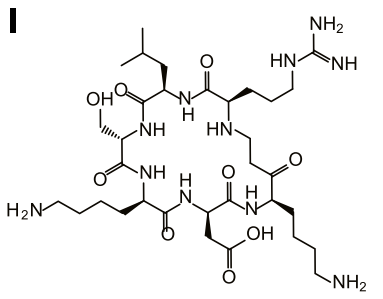

BK1361

$\left(\mathrm{IC}_{50}=120 \mathrm{nM} ;\right.$ ADAM-8)

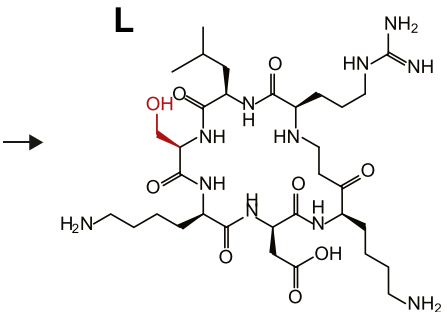

peptide 3

$\left(\mathrm{IC}_{50}=157 \mathrm{nM} ;\right.$ ADAM-8)

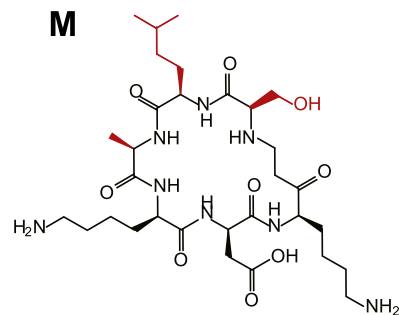

peptide 9

(IC 50220 nM; ADAM-8)

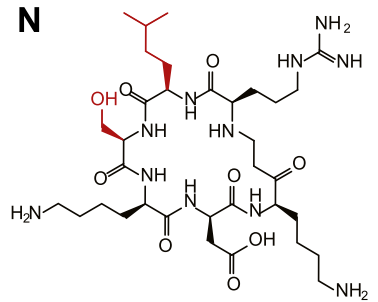

peptide 19

$\left(\mathrm{IC}_{50}=142 \mathrm{nM} ; \mathrm{ADAM}-8\right)$

FIGURE 1 | Peptide inhibitors of ADAM proteins. (A) Chemical structure of regasepin 1 linear peptide (PRC(Bip)CGE); (B) Chemical structure of regasepin 1-derived linear peptide (D-Pyr)-(D-Cys)-Bip-(D-Cys); (C) Chemical structure of peptide PL (Hisb-LPK-NH2); (D) Chemical structure of linear peptide Hxm-FRQ; (E) Chemical structure of linear peptide Hxm-FSN; (F) Schematically depicted structure of polycyclic octadecapeptide rhesus $\theta$-defensin-1 (RTD-1) peptide; (G) Schematically depicted structure of polycyclic octadecapeptide rhesus $\theta$-defensin-2 (RTD-2) peptide; (H) Schematically depicted structure of polycyclic octadecapeptide rhesus $\theta$-defensin-5 (RTD-5) peptide; (I) Chemical structure of cyclic peptide BK1361 (RLsKDK); (L) Chemical structure of cyclic peptide 3 (RLhS ${ }_{\beta}$ KDK); (M) Chemical structure of cyclic peptide $9\left(\mathrm{RL}^{*} \mathrm{~A}_{\beta} \mathrm{KDK}\right)$; (N) Chemical structure of cyclic peptide $19\left(\mathrm{RL}^{*} \mathrm{hS} \mathrm{\beta}_{\beta} \mathrm{KDK}\right)$. Indicated half maximal inhibitory concentration (IC $\left.{ }_{50}\right)$ and inhibition constant $(K \mathrm{~K})$ values were reported as published. The targeted $A D A M$ protein is reported near the $I_{50}$ or $K_{i}$ value. Legend: Bip $=$ biphenylalanine, $P y r=p y r i d y l a l a n i n e, ~ H i s b=(R)-2-$ isobutylsuccin hydroxamate moiety, $\mathrm{Hmx}=$ hydroxamate moiety, $\mathrm{S}=\mathrm{D}$-serine; $\mathrm{hS} \beta=\beta$-homoserine, $\mathrm{A}_{\beta}=\beta$-alanine, $\mathrm{L}^{*}=$ homoleucine. The side chains that differentiate from the parental peptide are shown in red.

(D-Pyr)-(D-Cys)-Bip-(D-Cys) (Figure 1B), showed an 8-fold higher potency $\left(\mathrm{IC}_{50}=600 \mathrm{nM}\right)$ than regasepin 1, 14-fold better selectivity against MMP-9 and 46-fold against MMP-3. However, no specificity for MMP-8 has been shown yet (Supplementary Table 1; (Qiu et al., 2012)). Similarly, Geurink and colleagues applied a synthetic combinatorial library of ninety-six enantiopure peptidomimetics bearing a zinc binding hydroxamate group at the $\mathrm{N}$-terminal and identified eight peptide variants capable of inhibiting ADAM17 with potencies in the sub-nanomolar to low micromolar range (Geurink et al., 2008). One peptide, named PL (Figure 1C), revealed an $\mathrm{IC}_{50}$ of $92 \mathrm{nM}$ against ADAM-17 and showed 40-fold better selectivity against MMP-9 but limited selectivity for MMP12 (Supplementary Table 1). By using a virtual combinatorial library of peptides derived from the TNF protease inhibitor 2 (TAPI-2), a broad-spectrum peptide inhibitor of ADAM-17 bearing a hydroxamate group, Wang and colleagues identified two linear peptides, named Hxm-Phe-Arg-Gln (Figure 1D) and Hxm-Phe-Ser-Asn (Figure 1E) that exhibited high potency toward ADAM-17 $\left(K_{\mathrm{i}}=47\right.$ and $92 \mathrm{nM}$, respectively $)$ and moderate selectivity toward ADAM-10 (5-fold and 7-fold, respectively; Supplementary Table 1; (Wang et al., 2016)). Furthermore, Schaal and colleagues discovered novel peptidebased inhibitors of ADAMs by screening $\theta$-defensins, a family of eighteen-amino acid macrocyclic peptides expressed exclusively in granulocytes and selected epithelia of Old World monkeys (Schaal et al., 2017)). The octadecapeptide rhesus $\theta$-defensin-1 (RTD-1) includes six disulfide-linked cysteines (Figure 1F) and inhibited ADAM-17 and ADAM-10 with an $\mathrm{IC}_{50}$ of 110 and $450 \mathrm{nM}$, respectively (Supplementary Table 1). Notably, RTD-1 showed at least 4-fold better selectivity toward a panel of related MMPs $\left(\mathrm{IC}_{50}=2-20 \mu \mathrm{M}\right.$; Supplementary Table 1$)$. When tested in vivo in a rodent model of rheumatoid arthritis, RTD-1 rapidly suppressed joint disease progression, restored limb mobility, and preserved normal joint architecture (Schaal et al., 2017). Further characterisation of five RTD isoforms (RTDs 1-5) revealed the presence of two macrocycles, RTD-2 (Figure 1G) and RTD-5 (Figure 1H), that inhibited ADAM-17 with $\mathrm{IC}_{50}$ values of 52 and $55 \mathrm{nM}$, respectively (Supplementary Table 1; (Schaal et al., 2018)). 


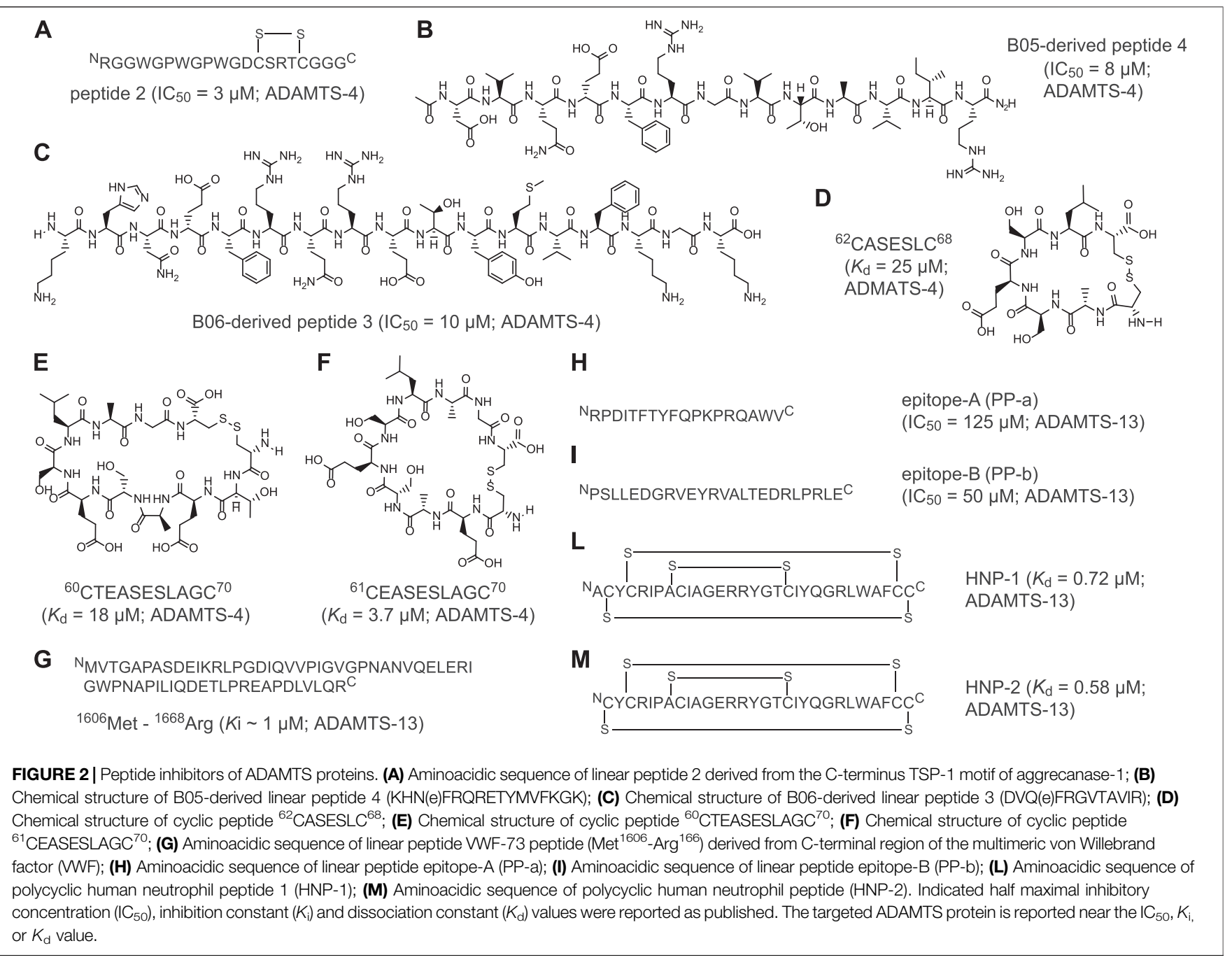

Another widely investigated sheddase is ADAM-8, a proteolytically active member of the ADAM protease family involved in numerous inflammatory processes (Schlomann et al., 2000) and neoplasia (Fritzsche et al., 2006; Romagnoli et al., 2014). By applying structural modeling and peptidomimetic approaches, Schlomann and colleagues generated a series of six amino acids cyclic peptides mimicking the RLSKDK motif of the mouse ADAM-8 (Schlomann et al., 2015). To enhance its potency and further increase its stability in vivo, the peptide sequence was constrained through cyclisation and modified with D-form amino acids in place of Arg, Leu, or Ser. The most potent cyclic peptide sequence contains a D-Ser ("s") (RLsKDK) (Figure 1I). The RLsKDK, also named BK-1361, inhibited ADAM-8 with IC $_{50}$ of $182 \mathrm{nM}$. Importantly, BK-1361 showed more than 100 -fold better selectivity toward other related metalloproteinases such as ADAM-9, -10, -12, -17 and MMP-2, -9, -14 (Supplementary Table 1). Notably, when tested in a mouse model of pancreatic ductal adenocarcinoma (PDAC), BK-1361 led to reduction of tumor load, infiltration and metastasis. Thus, further supporting the important role of ADAM-8 in PDAC development (Schlomann et al., 2015).
Additional structure-activity relationship studies on BK-1361 enabled the generation of eighteen structural analogue peptidomimetics (Yim et al., 2016). Among all tested cyclic peptides, peptides 3 (Figure 1L), 9 (Figure 1M) and 19 (Figure 1N) showed comparable or slightly higher inhibitory potency than the parental BK-1361 (Supplementary Table 1) (Yim et al., 2016).

\section{PEPTIDE-BASED INHIBITORS OF ADAMTSs}

The physiological function of ADAMTSs and their role in numerous pathologies have been described only recently (Zhong and Khalil, 2019; Apte, 2020). The first member of this family, ADAMTS-1, was characterised for the first time in 1997 (Kuno et al., 1997). Since then, few examples of peptide-based inhibitors against ADAMTSs have been reported (Tortorella et al., 2000; Hills et al., 2007; Di Stasio et al., 2008; Moriki et al., 2010; Pillai et al., 2016; Zhang et al., 2018). 
Recently, major efforts have been devoted to developing peptide-based inhibitors against two members of the ADAMTS family: ADAMTS-4 and ADAMTS-13 (Figure 2 and Supplementary Table 2). ADAMTS-4 cleaves proteoglycans such as aggrecan and versican, which play a structural role in many tissues (Fosang and Little, 2008). In fact, degradation of aggrecan is a clinical hallmark of degenerative joint disorders such as osteoarthritis Zhang et al. (2013), Yang et al. (2017) and rheumatoid arthritis (Mead and Apte, 2018). The first peptidebased inhibitors targeting ADAMTS- 4 were identified using linear peptides derived from the TSP-1 motif located at the C-terminus of the aggrecanase-1, an enzyme involved in cartilage degradation. The best selected peptide, peptide 2 (Figure 2A), showed an $\mathrm{IC}_{50}$ of $3 \mu \mathrm{M}$ against ADAMTS-4 (Supplementary Table 2; (Tortorella et al., 2000)). With the aim of identifying the cleavage motif of ADAMTS-4, Hills and co-workers applied phage display of random thirteen-amino acid linear peptide libraries to isolate seven-amino acid cleaved peptides with a wide range of potencies (Hills et al., 2007). Two linear peptides, B05 and B06, inhibited ADAMTS- 4 with potencies in the micromolar range $\left(\mathrm{IC}_{50}=35 \mu \mathrm{M}\right)$ and exhibited good selectivity toward the homologue ADAMTS-5 (Supplementary Table 2; Hills et al., 2007). Further studies revealed the importance of Glu in position P1 for substrate recognition and led to the development of novel synthetic peptides with modified stereochemistry of P1 and P1'. These two selected peptides inhibited ADAMTS- 4 with $\mathrm{IC}_{50}$ values of $8 \mu \mathrm{M}$ (peptide 4 , Figure 2B) and $10 \mu \mathrm{M}$ (peptide 3, Figure 2C) (Hills et al., 2007). Recently, Zhang and colleagues used computational modeling to develop peptide-based inhibitors from a loop of the N-terminal domain of TIMP3, a protein inhibitor of ADAMTS-4 (Zhang et al., 2018). Further peptide cyclisation diminished flexibility and enabled the generation of constrained molecules with reduced entropic penalty and improved binding affinities. Cyclised peptides ${ }^{62}$ CASESLC $^{68}$ (Figure 2D), ${ }^{61}$ CEASESLAGC $^{70}$ (Figure 2F) and ${ }^{60} \mathrm{CTEASESLAGC}^{70}$ (Figure 2E) showed bindings constants in the micromolar range $\left(K_{\mathrm{d}}=25 \mu \mathrm{M}, K_{\mathrm{d}}=3.7 \mu \mathrm{M}\right.$ and $K_{\mathrm{d}}=18 \mu \mathrm{M}$, respectively) and three- to 9 -fold increased affinity over the linear peptides (Supplementary Table 2).

In addition to ADAMTS-4, peptide-based inhibitors against ADAMTS-13 have been also developed. ADAMTS-13 is a metalloproteinase which cleaves the von Willebrand factor (VWF), a blood glycoprotein involved in haemostasis (Zheng, 2013). The levels of ADAMTS-13 correlate with ischaemic stroke risk, thrombotic thrombocytopenic purpura and microvascular thrombosis (South and Lane, 2018; Santamaria and de Groot, 2020). The first peptide-based inhibitor of ADAMTS-13 was identified by analysing the C-terminal region of the multimeric VWF factor, the VWF-73 peptide $\left(\mathrm{Glu}^{1660}\right.$ - $\mathrm{Arg}^{1668}$; Figure 2G) (Di Stasio et al., 2008). By elucidating the interaction of linear VWF-73 peptide with ADAMTS-13, Di Stasio and colleagues determined that inhibition occurs with a $K_{\mathrm{i}}$ value of $1 \mu \mathrm{M}$ (Supplementary Table 2). Furthermore, Moriki and colleagues applied phage display technology to identify two novel ADAMTS-13-derived peptide epitopes capable of binding VWF factor. Selected synthetic linear peptides PP-a and PP-b (Figures 2H,I) exhibited $K_{\mathrm{d}}$ values of 4.1 and $0.3 \mu \mathrm{M}$, respectively, and inhibited ADAMTS-13 with $\mathrm{IC}_{50}$ values of $125 \mu \mathrm{M}$ (PP-a) and $50 \mu \mathrm{M}$ (PP-b) (Supplementary Table 2) (Moriki et al., 2010). Finally, Pillai and co-workers showed that polycyclic human neutrophil peptides (HNP) inhibit the proteolytic cleavage of peptide VWF-73 and multimeric von Willebrand factor in a concentration-dependent manner. HNP-1 and -2 (Figures $2 \mathbf{L}, \mathbf{M}$ ) showed inhibitory concentrations in the low micromolar range and binding constants in the sub-micromolar range (Supplementary Table 2) (Pillai et al., 2016).

\section{CONCLUSIONS AND PERSPECTIVES}

ADAM and ADAMTS metalloproteinases play a significant yet complex role in several types of cancer, as well as in diverse inflammatory, neurodegenerative and cardiovascular diseases. Thus, a plethora of small chemical molecules and a few large proteins, such as monoclonal antibodies, have been developed to inhibit ADAM and ADAMTS metalloproteinases. While small chemical molecules often lack specificity and turn to be toxic, therapeutic proteins require high manufacturing costs and subcutaneous or intravenous administration. In this sense, peptide-based drugs offer a good alternative strategy with a surface of interaction large enough to obtain both high potency and selectivity, and yet small enough to diffuse into tissues. Other distinctive properties of peptides include chemical synthesis, ease of modification, low toxicity and reduced antigenicity. However, despite these favourable traits, peptides often have a relatively short circulating half-life and exhibit poor membrane permeability, which limit their broad applicability. While their systemic half-life can be prolonged by chemical conjugation to synthetic and natural polymers, or through non-covalent binding to endogenous proteins, such as serum albumin (Zorzi et al., 2019), reaching intracellular targets, on the other hand, is still a daunting task for peptide-based drugs. Although recent developments in chemical cyclization, methylation and the use of non-proteinogenic amino acids have led to promising results to overcome this problem, more accessible targets would also help to bypass the delivery strategies challenges (Cunningham et al., 2017; Lenci and Trabocchi, 2020). In this regard, ADAM and ADAMTS proteins have a peripheral extracellular localisation, which makes them ideal targets of peptide-based drugs. Moreover, the existence of multiple ADAM and ADAMTS homologues leverage the better selectivity of peptides (driven by their larger surface area and chiral complexity) over small-molecule drugs. The majority of linear and cyclic peptide inhibitors described in this mini review were developed using traditional medicinal chemistry approaches and structure-activity relationship studies on natural substrates and/or endogenous inhibitors. However, the specificity of some of the peptide inhibitors described here have not been fully investigated and none of them have reached a pre-clinical stage yet. Nevertheless, their development demonstrates that peptides represent valid molecular modalities for blocking the activity of ADAM and ADAMTS proteins. Indeed, the advent of novel DNA-encoded chemical libraries (Neri and Lerner, 2018) and superior peptide display technologies (Linciano et al., 2019; Sohrabi et al., 2020; Peacock and Suga, 2021) will enable the high-throughput screening of large combinatorial libraries, facilitating the discovery of novel potent and selective compounds with improved properties on short timescales (Sohrabi et al., 2020). Integration of these powerful 
combinatorial approaches with better automation, innovative chemical modification strategies and emerging computational methods will contribute to the development of better peptidebased inhibitors against ADAM and ADAMTS proteins, which have the potential to be used in the clinic in the near future.

\section{AUTHOR CONTRIBUTIONS}

SP, YM, and AA conceived the work. SP and YM analyzed data, prepared figures and wrote the first draft of the manuscript. AA supervised the work and edited the manuscript. All authors contributed to manuscript revision, read, and approved the submitted version.

\section{REFERENCES}

Apte, S. S. (2020). ADAMTS Proteins: Concepts, Challenges, and Prospects. Methods Mol. Biol. 2043, 1-12. doi:10.1007/978-1-4939-9698-8_1

Atangcho, L., Navaratna, T., and Thurber, G. M. (2019). Hitting Undruggable Targets: Viewing Stabilized Peptide Development through the Lens of Quantitative Systems Pharmacology. Trends Biochem. Sci. 44, 241-257. doi:10.1016/j.tibs.2018.11.008

Bakali, J. E., Gras-Masse, H., Maingot, L., Deprez, B., Dumont, J., Leroux, F., et al. (2014). Inhibition of Aggrecanases as a Therapeutic Strategy in Osteoarthritis. Future Med. Chem. 6, 1399-1412. doi:10.4155/FMC.14.84

Bessette, P. H., Rice, J. J., and Daugherty, P. S. (2004). Rapid Isolation of HighAffinity Protein Binding Peptides Using Bacterial Display. Protein Eng. Des. Sel. 17, 731-739. doi:10.1093/protein/gzh084

Cunningham, A. D., Qvit, N., and Mochly-Rosen, D. (2017). Peptides and Peptidomimetics as Regulators of Protein-Protein Interactions. Curr. Opin. Struct. Biol. 44, 59-66. doi:10.1016/j.sbi.2016.12.009

Daugherty, P. S. (2007). Protein Engineering with Bacterial Display. Curr. Opin. Struct. Biol. 17, 474-480. doi:10.1016/j.sbi.2007.07.004

Di Stasio, E., Lancellotti, S., Peyvandi, F., Palla, R., Mannucci, P. M., and De Cristofaro, R. (2008). Mechanistic Studies on ADAMTS13 Catalysis. Biophysical J. 95, 2450-2461. doi:10.1529/biophysj.108.131532

Dillen, C., Fiten, P., Chaltin, P., Hu, J., Dubois, V., Van den Steen, P., et al. (2006). Inhibition of Lethal Endotoxin Shock with an L-Pyridylalanine Containing Metalloproteinase Inhibitor Selected by High-Throughput Screening of a New Peptide Library. Cchts 9, 599-611. doi:10.2174/138620706778249758

Duffy, M. J., McKiernan, E., O'Donovan, N., and McGowan, P. M. (2009). Role of ADAMs in Cancer Formation and Progression. Clin. Cancer Res. 15, 1140-1144. doi:10.1158/1078-0432.CCR-08-1585

Edwards, D., Handsley, M., and Pennington, C. (2008). The ADAM Metalloproteinases. Mol. Aspects Med. 29, 258-289. doi:10.1016/j.mam.2008.08.001

Feldmann, M., and Maini, S. R. N. (2008). Role of Cytokines in Rheumatoid Arthritis: an Education in Pathophysiology and Therapeutics. Immunol. Rev. 223, 7-19. doi:10.1111/j.1600-065X.2008.00626.x

Fosang, A. J., and Little, C. B. (2008). Drug Insight: Aggrecanases as Therapeutic Targets for Osteoarthritis. Nat. Rev. Rheumatol. 4, 420-427. doi:10.1038/ncprheum0841

Fritzsche, F. R., Jung, M., Xu, C., Rabien, A., Schicktanz, H., Stephan, C., et al. (2006). ADAM8 Expression in Prostate Cancer Is Associated with Parameters of Unfavorable Prognosis. Virchows Arch. 449, 628-636. doi:10.1007/s00428006-0315-1

Georgiadis, D., and Yiotakis, A. (2008). Specific Targeting of Metzincin Family Members with Small-Molecule Inhibitors: Progress toward a Multifarious challenge. Bioorg. Med. Chem. 16, 8781-8794. doi:10.1016/j.bmc.2008.08.058

Geurink, P., Klein, T., Leeuwenburgh, M., Van Der Marel, G., Kauffman, H., Bischoff, R., et al. (2008). A Peptide Hydroxamate Library for Enrichment of Metalloproteinases: Towards an Affinity-Based Metalloproteinase Profiling Protocol. Org. Biomol. Chem. 6, 1244-1250. doi:10.1039/b718352f

Gilbert, A. M., Bikker, J. A., and O'Neil, S. V. (2011). Advances in the Development of Novel Aggrecanase Inhibitors. Expert Opin. Ther. Patents 21, 1-12. doi:10.1517/13543776.2011.539204

\section{FUNDING}

The authors declare that this study received funding from Fidia Farmaceutici S.p.A. The funder was not involved in the study design, collection, analysis, interpretation of data, the writing of this article or the decision to submit it for publication.

\section{SUPPLEMENTARY MATERIAL}

The Supplementary Material for this article can be found online at: https://www.frontiersin.org/articles/10.3389/fmolb.2021.703715/ full\#supplementary-material

Heinis, C., and Winter, G. (2015). Encoded Libraries of Chemically Modified Peptides. Curr. Opin. Chem. Biol. 26, 89-98. doi:10.1016/j.cbpa.2015.02.008

Hills, R., Mazzarella, R., Fok, K., Liu, M., Nemirovskiy, O., Leone, J., et al. (2007). Identification of an ADAMTS-4 Cleavage Motif Using Phage Display Leads to the Development of Fluorogenic Peptide Substrates and Reveals Matrilin-3 as a Novel Substrate. J. Biol. Chem. 282, 11101-11109. doi:10.1074/jbc.M611588200

Hu, J., Fiten, P., Van Den Steen, P. E., Chaltin, P., and Opdenakker, G. (2005a). Simulation of Evolution-Selected Propeptide by High-Throughput Selection of a Peptidomimetic Inhibitor on a Capillary DNA Sequencer Platform. Anal. Chem. 77, 2116-2124. doi:10.1021/ac048631p

Hu, J., Van Den Steen, P. E., Dillen, C., and Opdenakker, G. (2005b). Targeting Neutrophil Collagenase/matrix Metalloproteinase-8 and Gelatinase B/matrix Metalloproteinase-9 with a Peptidomimetic Inhibitor Protects against Endotoxin Shock. Biochem. Pharmacol. 70, 535-544. doi:10.1016/j.bcp.2005.04.047

Huang, Y., Wiedmann, M. M., and Suga, H. (2019). RNA Display Methods for the Discovery of Bioactive Macrocycles. Chem. Rev. 119, 10360-10391. doi:10.1021/acs.chemrev.8b00430

Huovila, A.-P. J., Turner, A. J., Pelto-Huikko, M., Kärkkäinen, I., and Ortiz, R. M. (2005). Shedding Light on ADAM Metalloproteinases. Trends Biochem. Sci. 30, 413-422. doi:10.1016/j.tibs.2005.05.006

Jackson, H. W., Defamie, V., Waterhouse, P., and Khokha, R. (2017). TIMPs: Versatile Extracellular Regulators in Cancer. Nat. Rev. Cancer 17, 38-53. doi:10.1038/nrc.2016.115

Kelwick, R., Desanlis, I., Wheeler, G. N., and Edwards, D. R. (2015). The ADAMTS (A Disintegrin and Metalloproteinase with Thrombospondin Motifs) Family. Genome Biol. 16, 113. doi:10.1186/s13059-015-0676-3

Kuno, K., Kanada, N., Nakashima, E., Fujiki, F., Ichimura, F., and Matsushima, K. (1997). Molecular Cloning of a Gene Encoding a New Type of MetalloproteinaseDisintegrin Family Protein with Thrombospondin Motifs as an Inflammation Associated Gene. J. Biol. Chem. 272, 556-562. doi:10.1074/jbc.272.1.556

Lambrecht, B. N., Vanderkerken, M., and Hammad, H. (2018). The Emerging Role of ADAM Metalloproteinases in Immunity. Nat. Rev. Immunol. 18, 745-758. doi:10.1038/s41577-018-0068-5

Lemarchant, S., Pruvost, M., Montaner, J., Emery, E., Vivien, D., Kanninen, K., et al. (2013). ADAMTS Proteoglycanases in the Physiological and Pathological central Nervous System. J. Neuroinflammation 10, 133. doi:10.1186/1742-2094-10-133

Lenci, E., and Trabocchi, A. (2020). Peptidomimetic Toolbox for Drug Discovery. Chem. Soc. Rev. 49, 3262-3277. doi:10.1039/d0cs00102c

Linciano, S., Pluda, S., Bacchin, A., and Angelini, A. (2019). Molecular Evolution of Peptides by Yeast Surface Display Technology. Med. Chem. Commun. 10, 1569-1580. doi:10.1039/c9md00252a

Malemud, C. J. (2019). Inhibition of MMPs and ADAM/ADAMTS. Biochem. Pharmacol. 165, 33-40. doi:10.1016/j.bcp.2019.02.033

Mead, T. J., and Apte, S. S. (2018). ADAMTS Proteins in Human Disorders. Matrix Biol. 71-72, 225-239. doi:10.1016/j.matbio.2018.06.002

Moriki, T., Maruyama, I. N., Igari, A., Ikeda, Y., and Murata, M. (2010). Identification of ADAMTS13 peptide sequences binding to von Willebrand factor. Biochem. Biophysical Res. Commun. 391, 783-788. doi:10.1016/j.bbrc.2009.11.138

Moss, M. L., and Minond, D. (2017). Recent Advances in ADAM17 Research: A Promising Target for Cancer and Inflammation. Mediators Inflamm. 2017, 1-21. doi:10.1155/2017/96735372017 
Moss, M. L., White, J. M., Lambert, M. H., and Andrews, R. C. (2001). TACE and Other ADAM Proteases as Targets for Drug Discovery. Drug Discov. TodayToday 6, 417-426. doi:10.1016/S1359-6446(01)01738-X

Murumkar, P. R., DasGupta, S., Chandani, S. R., Giridhar, R., and Yadav, M. R. (2010). Novel TACE Inhibitors in Drug Discovery: A Review of Patented Compounds. Expert Opin. Ther. Patents 20, 31-57. doi:10.1517/13543770903465157

Muttenthaler, M., King, G. F., Adams, D. J., and Alewood, P. F. (2021). Trends in Peptide Drug Discovery. Nat. Rev. Drug Discov. 20, 309-325. doi:10.1038/ s41573-020-00135-8

Neri, D., and Lerner, R. A. (2018). DNA-encoded Chemical Libraries: A Selection System Based on Endowing Organic Compounds with Amplifiable Information. Annu. Rev. Biochem. 87, 479-502. doi:10.1146/annurev-biochem-062917-012550

Peacock, H., and Suga, H. (2021). Discovery of De Novo Macrocyclic Peptides by Messenger RNA Display. Trends Pharmacol. Sci. 42, 385-397. doi:10.1016/ j.tips.2021.02.004

Pelay-Gimeno, M., Glas, A., Koch, O., and Grossmann, T. N. (2015). Structure-Based Design of Inhibitors of Protein-Protein Interactions: Mimicking Peptide Binding Epitopes. Angew. Chem. Int. Ed. 54, 8896-8927. doi:10.1002/anie.201412070

Pillai, V. G., Bao, J., Zander, C. B., McDaniel, J. K., Chetty, P. S., Seeholzer, S. H., et al. (2016). Human neutrophil peptides inhibit cleavage of von Willebrand factor by ADAMTS13: A potential link of inflammation to TTP. Blood 128, 110-119. doi:10.1182/blood-2015-12-688747

Plückthun, A. (2012). Ribosome Display: A Perspective. Methods Mol. Biol. 805, 3-28. doi:10.1007/978-1-61779-379-0_1

Qiu, Z., Yan, M., Li, Q., Liu, D., Van den Steen, P. E., Wang, M., et al. (2012). Definition of Peptide Inhibitors from a Synthetic Peptide Library by Targeting Gelatinase B/matrix Metalloproteinase-9 (MMP-9) and TNF- $\alpha$ Converting Enzyme (TACE/ADAM-17). J. Enzyme Inhib. Med. Chem. 27, 533-540. doi:10.3109/14756366.2011.599323

Raeeszadeh-Sarmazdeh, M., Do, L., and Hritz, B. (2020). Metalloproteinases and Their Inhibitors: Potential for the Development of New Therapeutics. Cells 9, 1313. doi:10.3390/cells 9051313

Rastogi, S., Shukla, S., Kalaivani, M., and Singh, G. N. (2019). Peptide-based Therapeutics: Quality Specifications, Regulatory Considerations, and Prospects. Drug Discov. Today 24, 148-162. doi:10.1016/j.drudis.2018.10.002

Romagnoli, M., Mineva, N. D., Polmear, M., Conrad, C., Srinivasan, S., Loussouarn, D., et al. (2014). ADAM 8 Expression in Invasive Breast Cancer Promotes Tumor Dissemination and Metastasis. EMBO Mol. Med. 6, 278-294. doi:10.1002/ emmm. 201303373

Santamaria, S., and de Groot, R. (2020). ADAMTS Proteases in Cardiovascular Physiology and Disease. Open Biol. 10, 200333. doi:10.1098/rsob.200333

Santamaria, S., and de Groot, R. (2019). Monoclonal Antibodies against Metzincin Targets. Br. J. Pharmacol. 176, 52-66. doi:10.1111/bph.14186

Santamaria, S., Fedorov, O., McCafferty, J., Murphy, G., Dudhia, J., Nagase, H., et al. (2017). Development of a Monoclonal Anti-ADAMTS-5 Antibody that Specifically Blocks the Interaction with LRP1. MAbs 9, 595-602. doi:10.1080/19420862.2017.1304341

Schaal, J. B., Maretzky, T., Tran, D. Q., Tran, P. A., Tongaonkar, P., Blobel, C. P., et al. (2018). Macrocyclic $\theta$-defensins Suppress Tumor Necrosis Factor- $\alpha$ (TNF- $\alpha$ ) Shedding by Inhibition of TNF- $\alpha$-Converting Enzyme. J. Biol. Chem. 293, 2725-2734. doi:10.1074/jbc.RA117.000793

Schaal, J. B., Tran, D. Q., Subramanian, A., Patel, R., Laragione, T., Roberts, K. D., et al. (2017). Suppression and Resolution of Autoimmune Arthritis by Rhesus $\theta$-defensin-1, an Immunomodulatory Macrocyclic Peptide. PLoS One 12, e0187868. doi:10.1371/journal.pone.0187868

Schlomann, U., Koller, G., Conrad, C., Ferdous, T., Golfi, P., Garcia, A. M., et al. (2015). ADAM8 as a Drug Target in Pancreatic Cancer. Nat. Commun. 6, 1-16. doi:10.1038/ncomms7175

Schlomann, U., Rathke-Hartlieb, S., Yamamoto, S., Jockusch, H., and Bartsch, J. W. (2000). Tumor Necrosis Factor a Induces a Metalloprotease-Disintegrin, ADAM8 (CD 156): Implications for Neuron-Glia Interactions during Neurodegeneration. J. Neurosci. 20, 7964-7971. doi:10.1523/jneurosci.20-21-07964.2000

Seegar, T. C., and Blacklow, S. C. (2019). Domain Integration of ADAM Family Proteins: Emerging Themes from Structural Studies. Exp. Biol. Med. (Maywood) 244, 1510-1519. doi:10.1177/1535370219865901

Simonetti, L., and Ivarsson, Y. (2020). Genetically Encoded Cyclic Peptide Phage Display Libraries. ACS Cent. Sci. 6, 336-338. doi:10.1021/acscentsci.0c00087
Sohrabi, C., Foster, A., and Tavassoli, A. (2020). Methods for Generating and Screening Libraries of Genetically Encoded Cyclic Peptides in Drug Discovery. Nat. Rev. Chem. 4, 90-101. doi:10.1038/s41570-019-0159-2

South, K., and Lane, D. A. (2018). ADAMTS-13 and von Willebrand factor: a dynamic duo. J. Thromb. Haemost. 16, 6-18. doi:10.1111/jth.13898

Sun, Y., Huang, J., and Yang, Z. (2015). The Roles of ADAMTS in Angiogenesis and Cancer. Tumor Biol. 36, 4039-4051. doi:10.1007/s13277-015-3461-8

Takeda, S. (2016). ADAM and ADAMTS Family Proteins and Snake Venom Metalloproteinases: A Structural Overview. Toxins 8, 155. doi:10.3390/toxins8050155

Tortorella, M. D., Tomasselli, A. G., Mathis, K. J., Schnute, M. E., Woodard, S. S., Munie, G., et al. (2009). Structural and Inhibition Analysis Reveals the Mechanism of Selectivity of a Series of Aggrecanase Inhibitors. J. Biol. Chem. 284, 24185-24191. doi:10.1074/jbc.M109.029116

Tortorella, M., Pratta, M., Liu, R.-Q., Abbaszade, I., Ross, H., Burn, T., et al. (2000). The Thrombospondin Motif of Aggrecanase-1 (ADAMTS-4) Is Critical for Aggrecan Substrate Recognition and Cleavage. J. Biol. Chem. 275, 25791-25797. doi:10.1074/jbc.M001065200

Wang, Z., Wang, L., Fan, R., Zhou, J., and Zhong, J. (2016). Molecular Design and Structural Optimization of Potent Peptide Hydroxamate Inhibitors to Selectively Target Human ADAM Metallopeptidase Domain 17. Comput. Biol. Chem. 61, 15-22. doi:10.1016/j.compbiolchem.2015.12.003

Wu, Y., Eigenbrot, C., Liang, W.-C., Stawicki, S., Shia, S., Fan, B., et al. (2007). Structural Insight into Distinct Mechanisms of Protease Inhibition by Antibodies. Proc. Natl. Acad. Sci. 104, 19784-19789. doi:10.1073/pnas.0708251104

Yang, C.-Y., Chanalaris, A., and Troeberg, L. (2017). ADAMTS and ADAM Metalloproteinases in Osteoarthritis - Looking beyond the 'usual Suspects'. Osteoarthritis and Cartilage 25, 1000-1009. doi:10.1016/j.joca.2017.02.791

Yim, V., Noisier, A. F. M., Hung, K.-y., Bartsch, J. W., Schlomann, U., and Brimble, M. A. (2016). Synthesis and Biological Evaluation of Analogues of the Potent ADAM8 Inhibitor cyclo(RLsKDK) for the Treatment of Inflammatory Diseases and Cancer Metastasis. Bioorg. Med. Chem. 24, 4032-4037. doi:10.1016/j.bmc.2016.06.042

Yiotakis, A., and Dive, V. (2008). Synthetic Active Site-Directed Inhibitors of Metzincins: Achievement and Perspectives. Mol. Aspects Med. 29, 329-338. doi:10.1016/j.mam.2008.06.001

Zhang, E., Yan, X., Zhang, M., Chang, X., Bai, Z., He, Y., et al. (2013). Aggrecanases in the Human Synovial Fluid at Different Stages of Osteoarthritis. Clin. Rheumatol. 32, 797-803. doi:10.1007/s10067-013-2171-0

Zhang, W., Zhong, B., Zhang, C., Wang, Y., Guo, S., Luo, C., et al. (2018). Structural Modeling of Osteoarthritis ADAMTS4 Complex with its Cognate Inhibitory Protein TIMP3 and Rational Derivation of Cyclic Peptide Inhibitors from the Complex Interface to Target ADAMTS4. Bioorg. Chem. 76, 13-22. doi:10.1016/ j.bioorg.2017.10.017

Zheng, X. L. (2013). Structure-function and Regulation of ADAMTS-13 Protease. J. Thromb. Haemost. 11, 11-23. doi:10.1111/jth.12221

Zhong, S., and Khalil, R. A. (2019). A Disintegrin and Metalloproteinase (ADAM) and ADAM with Thrombospondin Motifs (ADAMTS) Family in Vascular Biology and Disease. Biochem. Pharmacol. 164, 188-204. doi:10.1016/j.bcp.2019.03.033

Zorzi, A., Linciano, S., and Angelini, A. (2019). Non-covalent Albumin-Binding Ligands for Extending the Circulating Half-Life of Small Biotherapeutics. Med. Chem. Commun. 10, 1068-1081. doi:10.1039/c9md00018f

Zunke, F., and Rose-John, S. (2017). The Shedding Protease ADAM17: Physiology and Pathophysiology. Biochim. Biophys. Acta (Bba) - Mol. Cel Res. 1864, 2059-2070. doi:10.1016/j.bbamcr.2017.07.001

Conflict of Interest: SP is an employee of Fidia Farmaceutici S.p.A.

The remaining authors declare that the research was conducted in the absence of any commercial or financial relationships that could be construed as a potential conflict of interest.

Copyright (c) 2021 Pluda, Mazzocato and Angelini. This is an open-access article distributed under the terms of the Creative Commons Attribution License (CC BY). The use, distribution or reproduction in other forums is permitted, provided the original author(s) and the copyright owner(s) are credited and that the original publication in this journal is cited, in accordance with accepted academic practice. No use, distribution or reproduction is permitted which does not comply with these terms. 